Revue européenne des sciences sociales

European Journal of Social Sciences

XLIV-134 | 2006

Quel(s) défi(s) pour les sciences sociales à l'heure de la mondialisation?

\title{
Les sciences sociales et les défis de la mondialisation
}

\section{Giovanni Busino}

\author{
(2) OpenEdition \\ Journals \\ Édition électronique \\ URL : http://journals.openedition.org/ress/281 \\ DOI : $10.4000 /$ ress. 281 \\ ISSN : 1663-4446 \\ Éditeur \\ Librairie Droz \\ Édition imprimée \\ Date de publication : 1 juin 2006 \\ ISBN : 9-782-600-01095-5 \\ ISSN : 0048-8046
}

Référence électronique

Giovanni Busino, «Les sciences sociales et les défis de la mondialisation », Revue européenne des sciences sociales [En ligne], XLIV-134 | 2006, mis en ligne le 14 octobre 2009, consulté le 19 avril 2019. URL : http://journals.openedition.org/ress/281 ; DOI : 10.4000/ress.281 


\section{Giovanni BUSINO}

\section{LES SCIENCES SOCIALES ET LES DÉFIS DE LA MONDIALISATION}

Les termes mondialisation et son compère globalisation, au sens que nous leur attribuons de nos jours (antonymes d'harmonisation générale et d'ordre mondial), ont fait leur apparition dans le vocabulaire français dès la fin des années 1950 . D'emblée, ces termes ont désigné une situation de crise marquée par l'apparition de pouvoirs et de contraintes nouveaux (économiques, financiers, scientifiques, culturels) ainsi que par des cadres de vie inédits indiscernables et pourtant bien concrets, tels que le nivellement et l'uniformisation des phénomènes, le durcissement de la compétition économique, les inégalités des conditions de production, la circulation non maîtrisable des capitaux, l'hégémonie des biens profanes, les contacts rapprochés et asymétriques entre les cultures et les civilisations, la domination écrasante des savoirs techniques, les idéologies particularistes qui occultent les intérêts particuliers et les rapports de force' ${ }^{1}$.

Le terme mondialisation, qui évoque la perception et la description de cette situation de crise, en suggère aussi implicitement l'explication: la généralisation du système capitaliste, les mécanismes du marché mondialisé, le développement des transports, la suppression des distances grâce aux nouvelles technologies de l'information. Ces phénomènes créent des interdépendances, morcellent les solidarités, désagrègent les valeurs établies, valorisent les vertus de l'éclatement, écrêtent les différences, ébranlent les structures sociales, accentuent les processus d'exclusion et de marginalisation, rendent précaires les politiques publiques et impuissants les Etats nationaux.

Le remplacement de l'égalitarisme par le relativisme, la proclamation que toutes les cultures et toutes les expressions esthétiques sont légitimes, que tous les systèmes de valeurs sont équivalents ont contribué à amplifier les désarrois et à susciter des attitudes conformistes, des formes de ségrégation volontaire, des réactions de rejet de la rationalité, de la tolérance, de la non-violence, d'où le développement des particularismes et des mouvements ethniques, nationalistes, xénophobes et raciaux. Rorty affirme qu'une telle évolution vers le stade où nous n'adorerons «plus rien » a pour conséquence la dissémination de l'autorité et de la vérité, le remplacement des projets collectifs par la régulation procédurale ${ }^{2}$. D'où

Sur l'histoire du mot «globalisation», lire M. F. Leruth, «French Intellectuals come to terms with globalization », Contemporary French Civilisation, 25(1), 2001, p. 42-83: ainsi que G. Leclerc, La mondialisation culturelle: les civilisations à l'épreuve, Paris, PUF, 2000 et du même La société de communication: une approche sociologique et critique, Paris, PUF, 1999.

2 R. Rorty, Science et Solidarité: la vérité sans le pouvoir, Paris, Ed. de l'éclat, 1990. Pour une discussion de cette doctrine, cf. R. A. Kuipers, Solidarity and the Stranger: themes in the social philosophy of Richard Rorty, Lanham, Md., University Press of America, 1997. 
le sentiment de plus en plus diffus d'être victimes d'un monde inhumain et non maîtrisable.

Le sociologue sait que la perte des points de repère engendre des convictions floues, des désirs provisoires, une crise profonde de la subjectivité, de l'intériorité, de l'identité, du lien social, les incertitudes sur le sort personnel; il sait que dans ces conditions il est difficile d'accéder aux réalités universelles, aux significations des autres, d'admettre la pluralité des mondes, la pluralité et le partage des cultures; il constate également la multiplication des flux et des échanges, la mobilité imposée par l'espace marchand, l'affaiblissement des instruments de l'action politique efficace. Des sociologues réputés affirment que les Etats, dans la forme actuelle, ne peuvent pas faire face aux mutations contemporaines, que les citoyens ont délégué la liberté de choisir aux contrôleurs de la sphère marchande, qu'ils ont désappris l'usage de toutes les formes de liberté sauf celle d'acheter ${ }^{3}$. Mais peut-on conceptualiser la mondialisation comme s'il s'agissait d'un phénomène unique? Peut-on réduire les processus générés par la mondialisation à des indicateurs d'une crise de la modernité ou à des indices de sa fin? Est-ce que la mondialisation est un processus vraiment unique ou bien y a-t-il de multiples mondialisations qui s'impliquent les unes les autres? S'agit-il de formes encore rudimentaires de rationalisation dont les fins pourraient être la construction d'un monde où les différences et les oppositions conflictuelles seraient soumises à la norme éthique de la non-violence?

De quelle façon tous les «social scientists » ont-ils abordé ces questions et y ont-ils répondu, le cas échéant?

Il est notoire que dans le dernier quart du $\mathrm{XX}^{\mathrm{e}}$ siècle le paradigme de la correspondance entre les systèmes sociaux et les acteurs a été délaissé au profit des travaux sur les systèmes sans acteurs, sur les stratégies rationnelles en situations complexes et imprévisibles ainsi que des études sur les acteurs en contextes de crises, de risques, de décomposition des liens sociaux, ou encore des recherches sur les acteurs qui, au nom d'appartenances et d'héritages particularistes, rejettent les orientations et les représentations communes et privilégient la violence ou les replis identitaires.

Ce divorce entre les systèmes et les acteurs a fait que les analyses sociologiques sur la mondialisation/globalisation ont produit, jusqu'ici, des savoirs fragmentés, compartimentés. En plus, à défaut de catégories pertinentes, d'indicateurs mesurables, d'une modélisation conférant de l'ordre à des données multidimensionnelles, planétaires, la conceptualisation n'a pu être élaborée qu'en termes statiques d'intégration des marchés, des cultures, de l'information, de la communication, d'idéologies, de crise de la politique, et surtout d'internationalisation des capitaux, de flux financiers, ou de structure, d'organisation, d'état du monde, ou encore en termes de rupture, de réaction, d'effondrement des régulations sociales. Dans un cas, la mondialisation/globalisation est une manière de décrire le réel, d'observer les nouvelles sociabilités instaurées par les technologies de l'immatériel, de représenter les imbrications du mondial et du local; dans l'autre cas, elle est l'idéologie du libéralisme économique, d'un capitalisme déterritorialisé,

Z. Bauman, Globalization: the human consequences, Cambridge, Polity Press, 1998 (trad. franç. : Les coûts humains de la mondialisation, Paris, Hachette, 1999). Voir également J. Assayag, La mondialisation vue d'ailleurs : l'Inde désorientée, Paris, Seuil, 2005. 
soucieux de déréglementation, de compétitivité, de réactivité, de flexibilité, de marchés du travail polyvalent, de privatisation, d'avantages concurrentiels; elle est la cause principale de l'explosion de l'économie informelle, du chômage, de l'emploi précaire, du travail à domicile, de la sous-traitance.

Pour les uns, l'homogénéisation économique du monde et la croissance illimitée qui en découle combleront les différences d'équité et de droits entre les populations, tandis que, pour les autres, la régulation marchande de l'ordre social creusera les inégalités à l'intérieur de chaque pays, augmentera les clivages entre les sociétés du Nord et celles du Sud. Certains considèrent qu'il s'agit de la suite du vieux phénomène de l'internationalisation, du prolongement de mouvements séculaires, commencés au $\mathrm{XV}^{\mathrm{e}}$ siècle et puissamment accélérés aux $\mathrm{XIX}^{\mathrm{e}}$ et $\mathrm{XX}^{\mathrm{e}}$ siècles. D'autres proclament qu'on assiste à un bouleversement majeur de toutes les réalités sociales antérieures, voire à une modification singulière de notre être au monde ${ }^{4}$.

Peut-être que dans les années à venir les recherches comparatives et multidisciplinaires nous aideront à mieux cerner ces problématiques. En attendant, ici et maintenant, il faut utiliser les quelques synthèses disponibles à la façon de l'écrivain Jorge Luis Borges, qui reconstitue une civilisation perdue à l'aide de quelques livres d'une petite bibliothèque.

Déjà en 1966, Wilbert Ellis Moore, puis en 1980 Johan Vincent Galtung, en 1982 Niklas Luhmann, et en 1991 Anthony Giddens ont essayé d'esquisser les contours d'une sociologie de la mondialisation/globalisation, mais c'est à Immanuel Wallerstein, dont les premiers travaux remontent à 1979, et à Roland Robertson (ses recherches ont été réunies en un volume en 1992) que nous devons les études les plus méthodiques sur la mondialisation en tant que système sans acteurs ${ }^{5}$. Véritables paradigmes, au sens de Merton, ces études se fondent sur des hypothèses cohérentes et, pour certaines d'entre elles, vérifiables empiriquement.

\footnotetext{
${ }^{4}$ Un bon état de la question se trouve dans N. Fliegstein, «Rhétorique et réalités de la mondialisation", Archives de la recherche en sciences sociales, ${ }^{\circ} 119,1997$, p. 36-47. Voir aussi M. Albrow, The Global Age: State and Society Beyond Modernity, Cambridge, Polity Press, 1996; C. de Boissieu (dir.), Les mutations de l' économie mondiale, Paris, Economica, 2000; D. Held et al., Global Transformations: Politics, Economics and Culture, Cambridge, Polity Press, 1999; B. Holden (dir.), Global Democracy: Key Debates, London, Routledge, 2000; R. Kiely et P. Marfleet (dir.), Globalization and the Third World, London, Routledge, 1998; A. McGrew (dir.), The Transformation of Democracy? Globalization and Territorial Democracy, Cambridge, Polity Press, 1997; Y. Yamamoto (dir.), Globalism, Regionalism and Nationalism: Asia in Search of Its Role in the $21^{\text {th }}$ Century, London, Blackwell, 1999.

5 W. E. Moore, «Global Sociology. The World as a Singular System», American Journal of Sociology, vol. 71, n 5, 1966, p. 475-482; J. V. Galtung, The True Worlds: A Transnational Perspective, New York, Free Press, 1980; N. Luhmann, «The World Society, a Social System», International Journal of General System, VIII, 1990; A. Giddens, The Consequences of Modernity, Cambridge, Polity Press, 1991. I. Wallerstein, The Capitalist World-Economy, Cambridge, University Press, 1979; du même auteur: Historical Capitalism, London, Verso, 1984; «Societal Development or Development of the Modern World-System», International Sociology, n' I, 1986, p. 3-17; "Culture as the Ideological Battleground of the Modern World-System», dans M. Featherstone (dir.), Global Culture, Nationalism, Globalization and Modernity, London, Sage, 1990; Unthinking Social Science: The Limits of Nineteenth Century Paradigms, Cambridge, Polity Press, 1991; «The Rise and Future Demise of World-Systems Analysis », Review of Fernand Braudel Center, vol. I, n 21, 1998, p. 103-112. R. Robertson, Globalization. Social Theory and Global Culture, London, Sage, 1992, ainsi que le recueil d'articles édités par M. Featherstone, op. cit.
} 
Immanuel Wallerstein démontre qu'à partir du $X V^{\mathrm{e}}$ siècle s'est mis en place un capitalisme devenu, dès le $\mathrm{XVI}^{\mathrm{e}}$ siècle, une économie-monde. Des cycles longs alternant l'expansion et la stagnation, la récession et la reprise, ont bâti, au fil de cinq siècles, l'unité systémique de l'économie-monde. Les stagnations et les récessions ont été maîtrisées grâce à des processus de changements et de développements technologiques, à la prolétarisation des travailleurs et à l'incorporation de nouveaux territoires. Ces processus ont été consolidés et garantis par la généralisation des Etats nationaux, par les luttes entre les deux classes antagonistes, par la compétition entre les capitalistes. Cela explique la nature et la spécificité des nationalismes, des racismes, du sexisme, des idéologies religieuses, des tensions ethniques ainsi que de nombreuses autres réalités sociales. Les caractéristiques fondamentales de ce système sont la globalité et la tendance à l'autoconservation et à l'équilibre.

Le système de l'économie-monde capitaliste ne change pas de substance malgré les transformations des positions relatives des centres et des périphéries. Les mouvements antisystème sont les produits du système-monde lui-même, donc ils ne peuvent pas en modifier la nature ni les rôles. Les déséquilibres produits demeurent fonctionnels; ils participent au maintien de l'ordre systémique. Certes, le système n'est pas exempt de contradictions. A cause de leur déclin démographique et du vieillissement de leurs populations, les pays riches ont besoin de travailleurs africains et asiatiques, mais ils ne veulent pas leur reconnaître de véritables droits d'établissement. Alors que la multiplication des flux et des échanges économiques, à l'échelle planétaire, est sans limite, alors que dans la vie sociale les contraintes économiques internationales sont déterminantes, dans la vie nationale l'Etat contrôle toujours le centre et la périphérie. Il est le garant des inégalités internes et des échanges inégaux consubstantiels à la division internationale du travail. Il constitue la structure fonctionnelle indispensable à l'accumulation capitaliste, il est une création du système, sans quoi ni la genèse ni la reproduction de ce dernier ne seraient effectives.

La théorie du système-monde représente fort bien certains caractères structuraux de la mondialisation. Cependant, du fait qu'elle dérive d'un échange inégal, à la fois de la domination du centre sur la périphérie et de l'unité systémique du monde, cette théorie ne laisse pas la portion congrue au pouvoir, à la conquête, à la colonisation, aux multiples causes qui ont produit et reproduit l'échange inégal, notamment l'appropriation des surplus, la direction de leurs flux, la division du travail; elle méconnaît l'existence d'une dimension politique internationale antérieure aux dynamiques du capitalisme; elle néglige le fait que le passage de la régulation étatique des marchés à la mondialisation a été voulue par les Etats pour financer leur endettement sur le marché financier mondial. Ce dernier n'a qu'une seule référence: le prix, dont la «vérité » s'étend par delà des biens et des services reconnus comme marchands. Pour lui, il n'y a pas d'autres valeurs que celles de la rationalité marchande.

A l'opposé de l'économisme de Immanuel Wallerstein, se situe le culturalisme de Roland Robertson. Celui-ci présume que les processus en cours sont en train de rapetisser le monde, de faire de l'Humanité une société unique. Une culture autonome englobant les dynamiques politiques et socio-économiques est en train d'apparaître. Elle constitue le cadre de référence par lequel les couples homogénéité/hétérogénéité, intégration/désintégration, unité/diversité seront dépassés par 
la synthèse du global. Cette culture transcende l'unité société-Etat et se situe au-delà de la société-nation; elle produit des processus d'intégration (culturelle, normative, communicative, fonctionnelle) et de désintégration (des codes, des mémoires, des signaux, des langages et des pratiques sociales); elle valorise les diversités, les variétés et les richesses spécifiques. En même temps, elle suscite la résistance à l'ordre et aux contraintes. La multiplication des flux culturels engendre des tensions, mais elle favorise aussi l'élaboration des cultures transnationales, déterritorialisées, ouvertes aux échanges immatériels et aux contacts interpersonnels. Il y a des rencontres et des chocs entre les différentes cultures, mais ils prennent place à l'intérieur d'un contexte global, complexe, désordonné, lui-même en formation, en voie de construction.

Cette approche met en évidence la dynamique et l'autonomie des facteurs culturels, l'interdépendance de toutes les sociétés, mais surtout elle insiste sur le fait que les images, les représentations du monde sont construites et propagées aujourd'hui dans un contexte global. La globalisation est la conscience d'être dans un «lieu» qui n'est ni une communauté ni une société, lieu où les cultures, les sociétés, les individus adaptent leur existence particulière à l'ordre et au désordre d'un monde nouveau en formation.

Les composantes principales de cette construction théorique sont: l'Humanité, la citoyenneté, les droits de l'homme et le droit d'ingérence.

Par la référence à l'Humanité, l'individu peut dénoncer les violations du droit humanitaire, les destructions irréversibles de l'environnement, les risques sociaux et écologiques, les dangers pour la biodiversité, etc. Cette résistance relativise l'autonomie des sociétés particulières et l'hégémonie des Etats. Ces derniers ne peuvent plus faire abstraction des règles, des normes et des attentes du système mondial. La citoyenneté n'est plus un rapport privilégié entre l'individu et son Etat d'appartenance. Les migrations internationales en ont révélé la fragilité; elles marquent la contradiction entre cette appartenance particulière et l'appartenance universelle à l'espèce humaine. Les droits de l'homme ouvrent la voie au droit d'ingérence. La souveraineté et l'inviolabilité des Etats sont ainsi limitées. En bref, bien qu'elle se développe dans un contexte fluctuant et aux contours encore indiscernables, la globalisation favorise néanmoins la création d'une nouvelle image du monde; elle modifie la nature des identités et des cultures, leurs rapports réciproques; elle est inéluctable. Les renouveaux ou les renaissances des nationalismes, des ethnocentrismes, des fondamentalismes, des particularismes, ne sont que des moments « du double processus d'universalisation du particularisme et de particularisation de l'universalisme».

Contrairement aux théoriciens du système-monde qui attribuent ce double processus au capitalisme, Roland Robertson en fait une caractéristique de la nature humaine: l'universalisation du particularisme découle du fait que les hommes savent qu'il n'y a pas de limites à la spécificité, à l'unité, aux différences, à l'altérité des individus, des cultures, des sociétés, tandis que la particularisation de l'universalisme est la conséquence de la généralisation de l'idée de participer, quoique d'une position particulière, à une essence humaine universelle. Dans cette perspective, il n'y a pas de place pour le couple local/global. Les idées locales sont globalement vécues et propagées. Elles existent et s'affirment à l'aide et avec l'apport du contexte global. Ce processus serait bien représenté par le concept japonais de dochakuka, c'est-à-dire de «glocalisation», de localisation globale. 
A Anthony Giddens, pour qui la globalisation est la forme la plus radicale de la modernité, Roland Robertson oppose la nouveauté et l'originalité absolues du phénomène. Il ne s'agit pas d'une troisième révolution industrielle, mais d'une mutation aux conséquences exceptionnelles. Certes, l'Occident est le promoteur et le diffuseur d'idées et de concepts universels, et notamment du droit à la singularité, à l'autodétermination; certes, c'est l'Etat-nation, à l'origine société fermée, qui a maîtrisé et institutionnalisé les tensions hétérogènes internes. Il a obtenu la cohésion sociale avec l'invention de la tradition, avec l'imposition d'un certain degré d'homogénéisation sociale, avec la restriction des espaces ethniques, culturels, religieux, et il a fixé à tous des buts, par exemple le changement social et la modernisation. Ce rapport dialectique hétérogénéité/homogénéité est désormais devenu un rapport de type global/mondial. En conséquence, la diversité, le particularisme, le local, l'altérité, malgré la virulence de certaines revendications et contestations, coexistent avec l'unicité-uniformité du monde actuel et constituent les dimensions essentielles de la globalisation, des processus de mondialisation. L'étude d'une seule société ne permet pas de saisir ces réalités sociales nouvelles, ni les relatives «images du monde», ces constructions symboliques de la globalité produites par les processus en cours. D'où la nécessité pour la sociologie de changer d'orientation méthodologique, de délaisser l'analyse des sociétés locales et de devenir une sociologie internationale.

La théorie du système-monde et l'analyse culturaliste de la globalisation semblent foncièrement antagonistes, puisque la première privilégie les facteurs économiques et la seconde les aspects culturels et les formes de participation à l'unité de l'espèce humaine. Bien qu'il reconnaisse que certains processus de globalisation sont générés par les activités économiques, qu'ils contribuent à l'unification du monde, pour Roland Robertson les fondements de ces activités sont d'essence culturelle. C'est uniquement à la culture qu'il revient d'attribuer un sens à l'agir économique et de lui conférer une légitimité sociale. Pour cette raison, il est nécessaire d'inclure dans l'analyse de l'économie-monde les dynamiques culturelles produisant le développement de la conscience de la globalisation et les «images du monde» qui la représentent.

Ces deux orientations théoriques revitalisent la sempiternelle question de l'histoire de la sociologie, reformulée de façon attachante et perspicace par Immanuel Wallerstein en 1998, puis débattue âprement par d'autres au cours des mois suivants ${ }^{6}$. Ces deux orientations théoriques sont complémentaires. En effet, elles suggèrent qu'il faut donner une orientation nouvelle à la recherche sociologique, que celle-ci doit prendre en compte la complexité des phénomènes actuels. Dans cette perspective, l'une et l'autre systématisent des matériaux disparates, offrent des suggestions aux recherches à venir, proposent des approches intéressantes. Elles laissent, il va sans dire, des questions sans réponses. Si la mondialisation/globalisation est un phénomène unique, comment rendre compte des divers ordres de réalité, des mondes distincts? Est-ce que les événements tragiques du

- I. Wallerstein, «L'héritage de la sociologie, la promesse de la science sociale», Sociétés contemporaines, $\mathrm{n}^{\text {os }} 33-34,1999$, p. 159-194. Voir la réaction de R. Boyer, «Le paradoxe des sciences sociales: la vue d'un économiste 'dissident'», La sociologie contemporaine, vol. 47, n 4, 1999, p. $19-45$. 
Cambodge, de l'Afghanistan, de l'Irak, de la Tchétchénie, les cruautés ethniques ici et là, les génocides rwandais, les purifications ethniques en Bosnie, au Kosovo, la décomposition du Liberia, de la Somalie, le démembrement de la Yougoslavie, sont à imputer aux dynamiques capitalistes, au fait que le marché a imposé à tous ses lois, au détriment des spécificités socioculturelles? Si le chaos ne s'est pas généralisé, il doit bien y avoir d'autres formes de régulation, peut-être moins puissantes et moins apparentes, mais néanmoins agissantes.

La sociologie de la globalisation et des «images du monde» néglige le fait que le cosmopolitisme, la culture déterritorialisée, le tourisme international, le consumisme, la peur du nucléaire, les préoccupations écologiques, les droits de l'homme, sont des phénomènes avant tout occidentaux. La globalisation en tant que conscience d'une unité, d'un ordre mondial et d'une harmonisation générale reste, dans le contexte des relations actuelles des pouvoirs entre les différentes sociétés et cultures, une hypothèse à repenser.

L'internationalisation de la production, les économies informelles, les flux des échanges, le capitalisme de marché, la mobilité et la rotation des capitaux, les marchés instables, les frontières perméables, les entreprises à forte intensité de capital, la vulnérabilité des travailleurs sans qualification, le chômage, la marginalisation, l'individualisation des risques, créent assurément une interdépendance entre les hommes et une interpénétration des sociétés. Les contraintes géographiques et temporelles comprimées par les nouvelles technologies de l'information et de la communication font apparaître des processus de destruction et de recomposition, d'intégration et de désintégration, d'érosion de la plupart des mécanismes de régulation habituels. Naturellement, l'impact sur la structure sociale (inégalité, pauvreté, exclusion), sur le travail (chômage, fragmentation des activités, morcellement des statuts, précarité) est considérable. Mais il est imprudent de réduire ces phénomènes à la mise en œuvre par le mode de production de mécanismes pour parvenir à des fins ou d'en trouver l'explication dans des déterminismes gouvernés par les lois de fonctionnement du système capitaliste. Certains pays arrivent néanmoins à se développer en profitant de leurs avantages comparatifs, tandis que pour d'autres le creusement des inégalités est inexorable. Pourtant, aucun ne veut être exclu des flux transnationaux et tous rappellent que le marché est une construction politique et juridique, que les interactions entre les décisions économiques et politiques sont capitales, car ce sont elles qui fixent les règles de l'échange.

Le système de la mondialisation comprend d'innombrables acteurs. Il ne coordonne pas automatiquement leurs actions et celles-ci ne produisent pas des effets équivalents. Les chefs des entreprises multinationales agissent autrement que les gestionnaires des fonds de pensions et d'investissements, autrement que les représentants des actionnaires et des investisseurs des fonds d'épargne salariale. Leurs orientations et leurs finalités n'appartiennent pas au même ordre.

Il y a tout d'abord les Etats. La mondialisation est le résultat des décisions des gouvernements et des parlements; c'est la résultante de leurs politiques commerciales, sociales, financières et économiques. Sans les politiques de stabilisation macro-économique, sans la libéralisation des marchés, sans la privatisation des entreprises, sans le développement des infrastructures publiques, il n'y aurait pas d'économie de marché. Les Etats ont consolidé les marchés financiers en donnant la priorité aux taux d'inflation les plus bas, aux conséquences pouvant découler 
des niveaux des taux d'intérêt, en privilégiant les résultats tangibles et immédiats, en écartant toute action collective comportant une prise de risque, toute quête du bien commun à long terme. C'est grâce aussi à la politique de désinflation compétitive que les Etats européens ont pu aboutir à l'intégration de l'euro. L'histoire nous a appris que le capitalisme se soumet à n'importe quelle logique sociale puisqu'il sait que la valorisation et l'accumulation du capital ne sont possibles que s'il existe des règles et des normes, nationales et internationales. Ce sont les Etats, assistés par les organisations internationales contrôlées d'une manière ou d'une autre par les gouvernements (OMC, IOSCO, ISMA, ISO, FIM, Banque mondiale, OCDE), qui dans les faits élaborent des modèles normatifs, fixent les contraintes pour la coordination des actions, les font valoir (souvent assistés par des firmes d'audits et de consultants), les garantissent, les légitiment idéologiquement, avec ou sans l'aide du World Economic Forum de Davos. L'OMC est dominée davantage par les Etats que par les lobbies des multinationales. Ses décisions, prises par consensus, contraignent les pays riches à négocier avec les pays pauvres. Les premiers prétendent que l'OMC s'occupe uniquement des conflits commerciaux, les seconds veulent en étendre les compétences aux préférences sociales, à la compétion des systèmes sociaux, aux questions de santé, d'environnement, d'alimentation, des services publics.

Ce sont encore les Etats qui décident souverainement quand et comment abandonner ou alléger les dettes des pays pauvres, qui cantonnent entre des frontières symboliques les populations considérées comme inutiles et indésirables et les tiennent à distance économique et culturelle, à l'écart des processus d'homogénéisation. Ce sont toujours les Etats qui peuvent limiter les effets des fractures sociales, contenir la pauvreté, l'exclusion et la ségrégation. Mais aussi d'autres organisations gouvernementales, comme le BIT, l'OMS, l'UIT, l'OMI, etc., luttent contre les excès de ces politiques, du néolibéralisme, pour la défense des intérêts des pays faibles, pour la protection sociale, pour l'emploi et le développement durable, pour le commerce équitable, contre la traite des migrants, pour la régularisation des clandestins. Le Programme des Nations Unies pour le développement (PNUD) a calculé que les transactions financières sur le marché des changes dépassent un milliard de dollars par jour et qu'une taxe de $0,1 \%$ rapporterait quelque 150 milliards de dollars par an. Ce montant suffirait largement à l'éradication de la pauvreté dans le monde.

Les classes moyennes sont aussi des acteurs actifs politiquement. Elles sont favorables à la mondialisation, mais préoccupées de la montée des inégalités, de l'insécurité économique, de la remise en cause des systèmes de protection sociale, de l'augmentation de la clochardisation, de l'incivilité, soucieuses du ressentiment des exclus, de la criminalité quotidienne, des violences des désespérés, du terrorisme (du type de la secte Aum, des attentats d'Oklahoma, de New York, de Madrid, de Londres ou de l'Islamisme radical). Ces classes exigent une régulation, une gouvernance globale, des projets pour encadrer les politiques publiques, des actions pour agir sur le réel. Unies par la peur des risques, ces classes ont peu de significations existentielles en commun.

Dans cette même perspective se situent de nombreuses ONG et des représentants de la société civile, tels que le P7, Greenpeace, Amnesty International, ATTAC, ou encore les groupes de la galaxie spontanéiste, les manifestants de Seattle, de Washington et de Genova. Pour tous ces acteurs, les exigences 
d'ajustement et de compétitivité érodent les impératifs de la solidarité sociale et sont la cause du chômage, de la précarité, des inégalités, d'injustices innombrables, de l'augmentation de la délinquance, des violences, des peurs, des incertitudes, de la perméabilité des frontières, de la mise au pas des souverainetés nationales, de la «macdonaldisation» de la planète...

D'autres acteurs prennent une importance croissante: les juges, qui interviennent de plus en plus au-delà des limites des compétences territoriales juridiques; les experts scientifiques, qui imposent leur arbitrage dans les dossiers internationaux les plus divers; les gouverneurs des banques centrales, qui déterminent les choix des politiques monétaires; les agences de presse internationales, qui contrôlent les informations livrées au public.

La capacité de ces acteurs individuels et collectifs de dérégler le système, d'en modifier le fonctionnement, d'en détourner les buts, ne doit pas être sous-évaluée. Ces acteurs en réseaux transforment les rapports de pouvoir et les relations interpersonnelles. Sans leur présence et leur participation active, la mondialisation ne serait qu'une fatalité, et la sociologie, une science des déterminations plaquées, après coup, sur les réalités historiques. Ces acteurs sont en train de faire apparaître de nouvelles légitimités, d'imposer de nouveaux rapports de force, de déstabiliser de nombreux enchaînements macrosociologiques, de faire émerger le besoin d'un ordre mondial structuré par le droit, d'un droit humanitaire régissant la souveraineté des Etats et les activités d'un tribunal international des droits humains. Un nouvel espace public qui associe les nouveaux acteurs est en train de surgir et de se constituer de façon rapide et soutenue ${ }^{7}$.

C'est vrai, il n'y a pas encore un pouvoir mondial institutionnalisé, effectif; mais certains acteurs agissent dans des contextes complexes, interconnectés, interdépendants, et qui contribuent à l'émergence de modèles normatifs, fragiles et temporaires, et de réglementations multiples basées sur la logique de l'évitement des contradictions: des fréquences radio et télé aux voies aériennes, des télécommunications au trafic postal, des prescriptions contre les épidémies et les maladies infectieuses à l'assistance sanitaire et humanitaire, du prix équitable des médicaments à la normalisation des appareillages électriques. Les télécommunications numérisées, la robotique, l'informatique et les ordinateurs, Internet, les biotechnologies, la culture commune des sons et des images accélèrent, accroissent la mutuelle dépendance de toutes les activités humaines. Dépourvues des traditionnels liens de causalité linéaire, ces activités se présentent aux chercheurs sous des formes enchevêtrées et indémaillables. Le temps réel sollicite les émotions, l'absence de temps morts change l'imaginaire ainsi que les modalités d'appréhension du réel et la perception du travail. La technoscience, le couplage de l'informatique et des machines réduisent le nombre des travailleurs, favorisent les délocalisations et raccourcissent les temps de production des biens comme ceux des services. Les rapports sociaux en sont modifiés, l'espace bascule dans l'intemporalité ou la simultanéité, dans l'urgence du présent. Les logiques de l'immédiateté et de la connexité deviennent déterminantes. Nos capacités d'interprétation, de sélection et de réorganisation sont ébranlées, elles deviennent

M. Gauchet, «Quand les droits de l'homme deviennent une politique», Le débat, n 110, 2000, p. 258-288, et du même La condition politique, Paris, Gallimard, 2005. 
tributaires de l'émotion et de la simplification. Les manières de voir, de vivre et de penser en sont transmuées, d'autant plus qu'il est aléatoire de séparer la vie réelle de l'existence virtuelle, l'authenticité de la simulation, le vrai du faux. La surévaluation du présent porte à ignorer le passé et le futur. L'égoïsme le plus extrême surclasse le bien commun transgénérationnel. Privés de recul temporel, soumis aux urgences, notre manière de comprendre et d'agir subit des mutations fondamentales.

Devant un changement social si rapide, les identités se dissolvent, ainsi que les représentations qui fournissent les repères identificateurs et les vecteurs des interdits, des permissions, des espoirs, de la résignation ainsi que de la puissance. Même les identités sociales ne proviennent plus des groupes d'appartenance, mais des modes d'interaction avec de nombreux acteurs et différents groupes. Les interactions entre les individus priment sur toutes les formes de médiations collectives. Les identités des ayants droit anonymes, des bénéficiaires, des exclus, partageant les peurs et les risques mais n'ayant aucune signification en commun, ces identités ne sont plus stables et permanentes. On observe déjà des identités de substitution. Pour agir, pour poursuivre ses intérêts, il faut alors se redéfinir, se doter de nouvelles identités sociohistoriques, sources de sens, de stabilité, de légitimité. Dans le monde contemporain, l'identité culturelle, mieux l'identification culturelle (celle qui détermine les associations et les antagonismes, les alliances et les orientations pratiques), gagne de plus en plus en importance par comparaison aux autres dimensions de l'identité.

Bien que les référents sociaux demeurent fortement localisés, les antennes paraboliques font tout vivre en temps réel et rendent le présent éternel. Elles diffusent partout la même musique, les mêmes séries télévisées, les mêmes films, les mêmes scènes de la quotidienneté. Inévitables, donc, sont les emprunts, les imitations, l'uniformisation des besoins et des attentes, l'harmonisation de plusieurs aspects des modes de vie. Inévitables aussi, les comparaisons entre ce que nous sommes et ce que sont les autres. Inévitables, les convergences des questionnements et des revendications à propos de la perte de crédibilité de la classe politique, de la corruption, à propos des conséquences perverses de la régulation, de l'étiolement de la cohésion sociale. Partout, même dans les contrées les plus isolées, on peut observer l'émergence d'une société civile, la présence d'acteurs qui revendiquent les droits de l'homme, qui reconstruisent, sans soucis idéologiques et normatifs, leurs différences, leurs particularismes, et puis les transposent au niveau global, comme si la mondialisation était la seule voie de reconnaissance et de légitimation de leurs aspirations.

La mondialisation est un phénomène complexe, multidimensionnel, qui remarie le système avec les acteurs. En tant que marché, organisation de la production des biens et des services, en tant que mécanisme de valorisation et d'accumulation du capital, elle décloisonne les frontières, libéralise les échanges, fait circuler librement les capitaux, sans aucunement tenir compte des attentes des salariés. Dans certains cas, elle met en cause le conservatisme catégoriel et contribue ainsi à moderniser les rapports sociaux. Elle est aussi l'idéologie qui voudrait imposer et légitimer la régulation marchande de l'ordre mondial.

Cependant, tous les phénomènes contemporains ne peuvent pas être expliqués par la mondialisation économique. Il est hasardeux de lui imputer la responsabilité de nos malheurs sociaux, d'en faire le pouvoir maléfique par essence. Il existe 
de multiples mondialisations et globalisations qui s'impliquent les unes les autres, qui touchent des ordres de réalité divers et distincts: le sport, les divertissements, le tourisme, la médecine, la science, le droit, le crime organisé, l'enseignement, le milieu des médias, etc. Il est ici superflu d'analyser toutes ces mondialisations. Il suffit de rappeler que ces mondes distincts utilisent les mêmes technologies de l'information et de la communication et que ces dernières véhiculent un monde où tout se passe instantanément et où la réflexion et l'analyse sont remplacées par les habitudes, les instincts et les émotions. Simples moyens vides de finalités, ces technologies ne véhiculent aucun projet politique précis, elles ne dictent pas les modalités d'organisation de leur emploi; elles ne transforment pas automatiquement l'individu et la société, ne les soumettent pas à une logique systémique. En faire des croyances, une idéologie de l'action au service d'enjeux économiques, un moyen de renforcer l'emprise des agents œuvrant à l'expansion du modèle capitaliste est dans l'ordre des choses. Mais ces technologies servent aussi à subvertir les hiérarchies pyramidales, à bloquer les initiatives, à rééquilibrer les rapports hommes-femmes, société civile-Etat autoritaire, ONG-parti unique, ou encore à résister à certaines valeurs et normes, à s'opposer à la coexistence de différents niveaux culturels dans un lieu déterminé, à détruire le lien social qui relie l'un à l'autre. Suppriment-elles les expériences humaines directes, rendent-elles cohérentes les convictions? Oui, lorsque l'activité médiatisée par l'outil est standardisée; non, lorsque l'activité est complexe et nécessite une forte dose d'invention et d'interactivité. Ces technologies ne sont pas définissables de façon unique. Caractérisées d'une incertitude objective, elles ne véhiculent ni des orientations ni des significations précises.

Il existe une idéologie de la mondialisation qui accouche d'une pensée unique, mais qui s'accommode sans états d'âme des diversités, des pluralismes, des complexités. Cette idéologie proclame l'universalisme, mais pratique un relativisme adéquat à tous les systèmes de valeurs et à toutes les situations historiques particulières. D'autre part, les valeurs au fondement de la mondialisation ne s'imposent pas à tout sujet, quel qu'il soit. Elles sont soumises, à partir d'un répertoire culturel et historique donné, à des modes d'appropriation, de réinvention, d'ajustement. Les valeurs traditionnelles sont consolidées par ces réinterprétations et réinventions. En effet, des représentations traditionnelles véhiculent le modèle capitaliste et l'économie de marché dans certains pays d'Asie et d'Afrique. Là où il y a rejet, réactions identitaires, conflits, on invoque et proclame les principes fondamentaux de l'universalisme (l'autodétermination, les droits de la personne, la spécificité de la culture), plus au titre de l'émotif et de l'affectif, de fins dispersées et incertaines, qu'à celui de la raison et de la hiérarchie des choses.

Amartya Sen essaie de redéfinir les fondements de ce nouvel universalisme en mettant l'accent sur les capacités fondamentales des personnes, sur leurs droits aux biens fondamentaux, sur le pouvoir de les réclamer, sur la liberté d'option, sur la situation historique particulière, sur la particularité qu'impose la condition humaine inscrite dans le temps historique.

Le sociologue peut aisément réinscrire les acteurs dans le système, reconnaître les multiples mondialisations qui s'impliquent les unes les autres, distinguer entre la mondialisation en tant qu'idéologie et ses pratiques effectives. Les difficultés majeures viennent du fait que dans les multiples mondialisations les fins ainsi que les représentations finalisées ne sont détectables que sur une très longue durée. A 
courte échéance, le sociologue peut observer les moyens, leur efficacité relative, les justifications de l'agir, les repères instables et incertains à la base de convictions floues, des rejets ou des adoptions, la crise de la subjectivité, de l'intériorité, de l'identité, du lien social. Dès lors, à défaut d'indicateurs symboliques et spatiaux, de valeurs collectives valables pour tous, ses généralisations, ses classements, ses systématisations ne l'aident pas à établir si les mondialisations sont porteuses de contradictions fondamentales ou de convergences possibles, si à travers les reformulations des enjeux et des institutions elles préludent à l'intégration, à une rationalité fondée sur la compatibilité des signifiances, ou si elles visent à fonder exclusivement une normativité tournée vers les pratiques quotidiennes ${ }^{8}$.

Envahis que nous sommes par le présent immédiat, avec ses dangers et ses incertitudes, ses violences et ses misères, nous avons oublié l'histoire et perdu le futur. Il est temps de revenir au projet primordial de la sociologie, selon lequel les sociétés se nourrissent de diversités de conflits, de valeurs et de symbolisations; les choix dictés par les désirs et les intérêts sont réversibles et éphémères, alors que ceux inspirés par les enracinements, les mythes, les espérances, les valeurs sont plus durables et contraignants. L'histoire nous montre que les sociétés peuvent trouver les moyens de réguler les conflits et de les rendre productifs, qu'elles savent le faire et pourraient le faire aujourd'hui'. La politique et la démocratie sont les instruments pour réaliser un monde commun habitable, partageable, conciliant les cultures, les aidant à voir ce qui leur est extérieur, à reconnaître les différences, à respecter l'éthique de la non-violence.

J'emprunte la conclusion de cet article trop rhapsodique à mon ami Jean Starobinski qui, dans le très bel essai «La présence au monde ${ }^{10}$, a écrit ce qui suit:

L'éthique de la non-violence [...] n'engage aucun individu à en rabattre sur son désir de l'universel ou de l'absolu, ni à sacrifier en rien les moyens figurés (et si souvent métaphoriques) à travers lesquels il l'appréhende.

Elle consiste à reconnaître le lieu particulier - le monde actuel et concret - à partir duquel nous formulons cette visée de l'universel, et à reconnaître que d'autres visées que la nôtre partent d'un autre lieu particulier, d'une autre existence incarnée. L'universel se réalise dans la reconnaissance commune de la particularité qu'impose notre condition, par quoi notre vie s'inscrit dans le temps historique. Une telle reconnaissance signifierait l'abolition du conflit sans l'abolition des identités. Nous en sommes éloignés, certes, mais cela n'interdit pas d'y penser et d'en faire une norme.

Genève

${ }^{8}$ Cfr. G. Busino, «Teoria della cultura», in Enciclopedia del Novecento, XII. Terzo Supplemento: Acqua-Guerra, Roma. Istituto dell’Enciclopedia Italiana, 2004, p. 305-313

9 R. Axtmann, «Society Globalization and the Comparative Method», History of the Human Sciences, vol. 6, n², 1993, p. 53-74.

10 J. Starobinski, «La présence au monde», dans Incertaine planète. Textes des conférences et des entretiens organisés par les trente-cinquièmes Rencontres internationales de Genève 1995, BoudryNeuchâtel, A la Baconnière, 1996, p. 15-32. 